\title{
Beyond the periodic orbit theory
}

\author{
Predrag Cvitanović, Kim Hansen, Juri Rolf and Gábor \\ Vattay 目 \\ Center for Chaos and Turbulence Studies \\ Niels Bohr Institute \\ Blegdamsvej 17, DK-2100 Copenhagen $\varnothing$, Denmark
}

\begin{abstract}
The global constraints on chaotic dynamics induced by the analyticity of smooth flows are used to dispense with individual periodic orbits and derive infinite families of exact sum rules for several simple dynamical systems. The associated Fredholm determinants are of particularly simple polynomial form. The theory developed suggests an alternative to the conventional periodic orbit theory approach to determining eigenspectra of transfer operators.
\end{abstract}

PACS numbers: 0320, 0365, 0545

AMS classification scheme numbers: $58 \mathrm{~F} 20$

Dedicated to Adrian Douady for his 60th birthday

\section{Introduction}

Low dimensional chaotic classical and quantum dynamical systems [1, 2] can be analyzed in terms of unstable periodic orbits. The periodic orbit theory of such systems has been successfully applied to a wide range of physical problems [3, 4, 5. However, since the phase space is tessellated into linearized neighbourhoods of periodic points, analyticity properties of smooth flows are not fully utilized in the conventional periodic orbit theory.

In this paper we explore global constraints on chaotic dynamics induced by the analyticity of the flow. We propose to dispense with the periodic orbits altogether, and extract spectra of transfer operators from dynamics-induced relations among periodic orbit sums.

$\ddagger$ Permanent address: Dept. Solid State Physics, Eötvös University, Muzeum krt. 6-8, H-1088 Budapest 
For example, evaluating trace formulas for 2-dimensional Hamiltonian flows [6] we have found exact periodic orbit sum rules

$$
\begin{gathered}
\sum_{i \in \operatorname{Fix}\left(f^{n}\right)} \frac{1}{\Lambda_{i}\left(1-1 / \Lambda_{i}\right)^{2}}=0 \\
\sum_{i \in \operatorname{Fix}\left(f^{n}\right)} \frac{\Lambda_{i}+1 / \Lambda_{i}}{\Lambda_{i}\left(1-1 / \Lambda_{i}\right)^{2}}=2^{n},
\end{gathered}
$$

where $f$ is a volume preserving Hénon map and $\Lambda_{i}$ is the expanding eigenvalue of its $i$ th periodic point, real or complex. Such relations are remarkable insofar they require a high degree of correlation between the stabilities of exponentially large numbers of periodic orbits distributed over the entire phase space. As a matter of fact, for at least two of the dynamical systems studied here, the quadratic polynomial map and the Farey map, the information carried by the periodic points is so redundant, that the periodic points must satisfy infinitely many independent sum rules.

The key idea behind the method by which the sum rules are derived is to replace a weighted sum over periodic orbits by an integral representation; the dynamics then induces recursion relations between these for cycles of different topological length. In posing the problem this way we draw inspiration from the work of Eremenko, Levin, Sodin, and Yuditskii [7, 8, 9] and Hatjispiros and Vivaldi [10 on the polynomial mappings, and Contucci and Knauf [1] for the Farey map. For these models the recursions relate finite numbers of terms, and yield expressions (27), 61) for the corresponding Fredholm determinants in terms of finite polynomials of form

$$
\operatorname{det}(1-z \mathcal{L})=\operatorname{det}(1-z \mathbf{L})
$$

where $\mathcal{L}$ is a weighted transfer operator and $\mathbf{L}$ is the corresponding finite $[\ell \times \ell]$ dimensional matrix that relates $\ell$ consecutive sums.

While this finiteness is a very special property of the particularly simple models considered and cannot hold for a generic dynamical flow, the method nevertheless suggests that one might be able to dispense with the periodic orbit theory altogether, and manipulate instead the traces of dynamical transfer operators by means of such integral representations. This tempting possibility motivates us to explore the applicability of the method in some depth, the main results being the sum rules and closed form expressions for Fredholm determinants of weighted transfer operators.

We start with polynomial mappings in section 3, and then specialize to quadratic mappings (section 1 ) for which an infinity of sum rules is easily derived. Special examples are worked out in the appendices. In section 5 we generalize a result of ref. [8] about the spectrum of a non-polynomial transfer operatorfor quadratic mappings. In section 6 we show that our method can be extended to higher dimensions and apply it to the Hénon map and the kinematic dynamo model. Finally, in order to show that the contour integrals are not the essence of the method, in section 7 we derive the same kind of explicit polynomial Fredholm determinants for the circle-map (or spin-chain) 
thermodynamics. We discuss the applicability of the method to continuous flows in section 2.1: the sum rules offer a new invariant characterization of the errors due to approximating a flow by a polynomial Poincaré section return mapping. The sum rules of section 1 use a "signed" rather than the "natural" measure; in section 8.2 we outline how they might be applicable to the period-doubling presentation function, for which the "signed" measure yields a formula for the Feigenbaum $\delta$. We finish with a critical summary and relegate some details to appendices.

\section{Transfer operators and Fredholm determinants}

We start with a brief summary of the dynamical systems concepts needed in what follows. For motivation and background we refer the reader to literature, for example refs. [12, 13].

A dynamical flow is usually investigated via its Poincaré return map $f$, with the evolution of a density function $\phi(x)$ given by

$$
(\mathcal{L} \phi)(y)=\int d x \delta\left(x-f^{-1}(y)\right) \phi(x)=\sum_{x: f(x)=y} \phi(x)
$$

(for the time being we assume that $f$ is a 1-dimensional map). A variety of applications [14, 12], some of which we shall turn to below, requires use of generalized transfer operators weighted by integer powers of the stability of the orbit

$$
\mathcal{L}_{(k)}(y, x)=f^{\prime}(x)^{k-1} \delta\left(x-f^{-1}(y)\right) .
$$

Let $f^{n}(x)$ be the $n$th iterate of the map, and

$$
\Lambda_{i}:=\prod_{j=0}^{n-1} f^{\prime}\left(f^{j}\left(x_{i}\right)\right)
$$

be the linear stability evaluated at the periodic point $x_{i}$, given by the product over the $n$ periodic points belonging to a given $n$-cycle. We shall denote the $n$th iterate raised to $m$ th power by $f^{n}(x)^{m}$, and the derivative of the $n$th iterate by $f^{n^{\prime}}(x)=\frac{d}{d x} f^{n}(x)$. Traces of the powers of this transfer operator are given by

$$
\operatorname{tr} \mathcal{L}_{(k)}^{n}=\sum_{x_{i} \in \operatorname{Fix}\left(f^{n}\right)} \frac{\Lambda_{i}^{k-1}}{\left|1-1 / \Lambda_{i}\right|},
$$

where we have assumed that all cycles have stability eigenvalues $\Lambda_{i} \neq 1$ strictly bounded away from unity. A cycle is called attracting, neutral or repelling if $\left|\Lambda_{i}\right|<1$, $\left|\Lambda_{i}\right|=1,\left|\Lambda_{i}\right|>1$, respectively. If the map is repelling and all $\left|\Lambda_{i}\right|>1$, we can drop the absolute value brackets in (四) and define the trace by

$$
T_{n}(k):=\sum_{x_{i} \in \operatorname{Fix}\left(f^{n}\right)} \frac{\Lambda_{i}^{k}}{\left(\Lambda_{i}-1\right)} .
$$


A corresponding "determinant" or "Zeta function" is related to the traces by

$$
F(z, k)=\operatorname{det}\left(1-z \mathcal{L}_{(k)}\right)=\exp \left(-\sum_{n=1}^{\infty} \frac{z^{n}}{n} T_{n}(k)\right)=\sum_{n=0}^{\infty} a_{n}(k) z^{n},
$$

and the spectrum of the transfer operator is given by the zeros of $F(z, k)$. In applications [14, 12] determinants are often preferable to the trace sums. For a lack of a better term we shall refer to this function as the "Fredholm determinant" of the transfer operator (2) even though, strictly speaking, the term should be used only for determinants of operators proven to be trace class.

The main difference between the generalized transfer operators defined by (2), and the operators that usually arise in the dynamical systems theory is that instead of the "natural measure" $1 /\left|\Lambda_{p}\right|$ we deal here with a "signed" and possibly complex cycle weight $1 / \Lambda_{p}$. There are several important applications of transfer operator formalism based on such "signed" measures, in particular the periodic orbit formulas for the spectrum of the period-doubling operator, section 8.2 .

From now on we define the trace of an transfer operator $\mathcal{L}$ by the above trace sum (5) over all cycles, real and complex. This choice of the cycle weight is motivated by the contour integral sums to which we turn now.

\section{Contour integral evaluation of periodic orbits sums}

A sum that runs over zeros of a function can be cast as a contour integral residue calculation. Periodic orbit formulas are sums over zeros of the periodicity condition $x-f^{n}(x)=0$, therefore we consider contour integrals of type

$$
T=\oint \frac{d x}{2 \pi i} \frac{h(x)}{f(x)-x}
$$

where $f$ and $h$ are polynomials, and the contour encloses all zeros of $f(x)-x$. By shrinking the contour to a sum of contours around individual zeros, figure f, and using the Cauchy formula

$$
\oint \frac{d x}{2 \pi i} \frac{1}{x^{k}}=\delta_{k, 1}
$$

we obtain a sum over fixed points $x_{i}$ of the map $f$ :

$$
T=\sum_{x_{i} \in \operatorname{Fix}(f)} \frac{h\left(x_{i}\right)}{f^{\prime}\left(x_{i}\right)-1} .
$$

Note that this cycle sum includes all periodic points, real as well as complex. For $f^{n}$, $n \rightarrow \infty$ these points fill out the Julia set, the closure of the set of the repelling periodic points 15. We assume that all zeros are simple; multiple zeros can be treated as well. Here we shall compute

$$
T(k)=\oint \frac{d x}{2 \pi i} \frac{f^{\prime}(x)^{k}}{f(x)-x}
$$




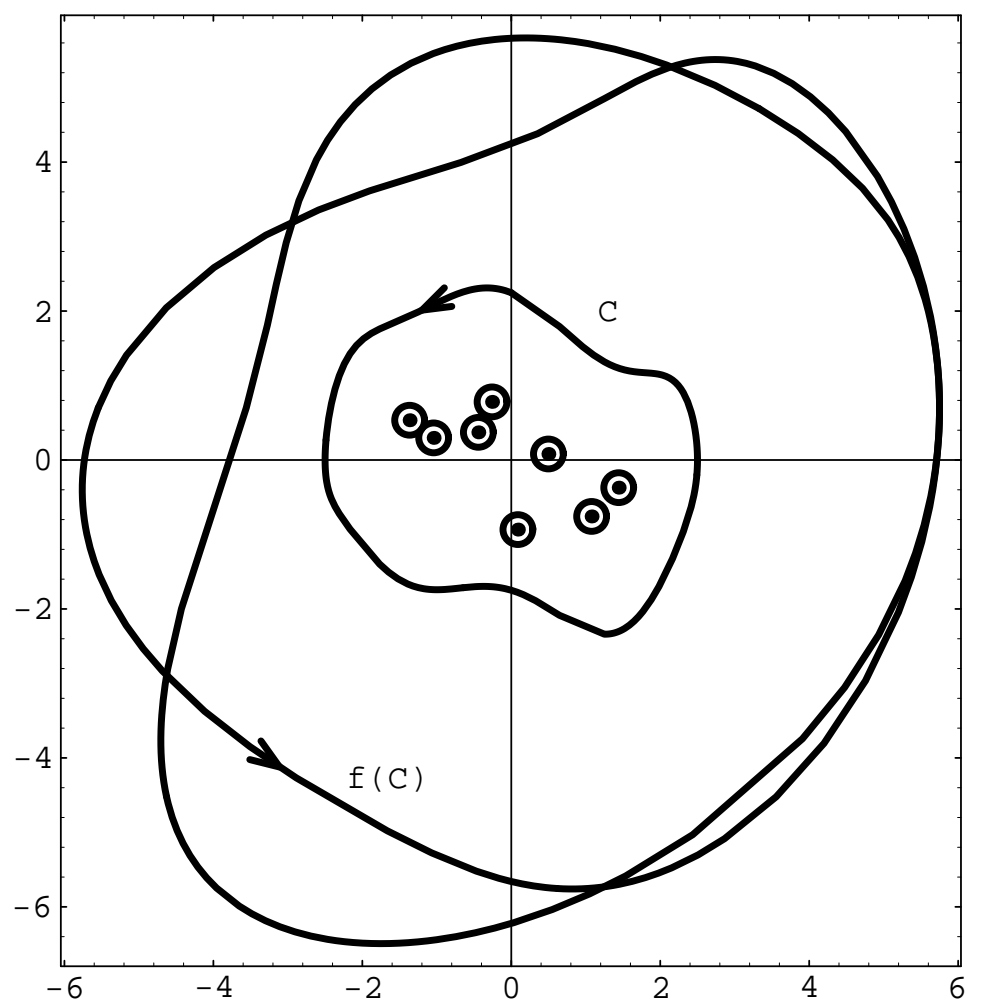

Figure 1. Shrinking the contour $C$ to a sum of contours around individual zeros leads to a sum over fixed points $x_{i}$ of the map $f$. For a sufficiently large contour $C$, the iterate $C \rightarrow f(C)$ wraps twice around the periodic point set, leading to the key formula (24). In this figure the map is $f(x)=x^{2}-c, c=0.5-0.7 i$, and the 8 periodic points plotted are the zeros of $f^{3}(x)-x=0$.

for $k=0,1,2,3, \ldots$ (we turn to the $k=-1$ case in section 5 ).

The key observation is that $T$ can be evaluated by pushing the contour to $|x| \rightarrow \infty$, where the individual fixed points play no role. When $k>0$, the following expansion of (8) is convenient:

$$
\begin{aligned}
T(k) & =\oint \frac{d x}{2 \pi i} \frac{f^{\prime}(x)^{k}}{x} \frac{x / f(x)}{1-x / f(x)} \\
& =\oint \frac{d x}{2 \pi i} \frac{f^{\prime}(x)^{k}}{x}\left(\frac{x}{f(x)}+\left(\frac{x}{f(x)}\right)^{2}+\cdots+\left(\frac{x}{f(x)}\right)^{k}\right) .
\end{aligned}
$$

For a polynomial this is an exact formula; we use the fact that for sufficiently large $|x|$ and order $N \geq 2$ of the polynomial $f,(1-x / f(x))^{-1}$ can be expanded as a geometric series. Terms with powers of $(x / f(x))^{r}$ higher than $r>k$ are of order at least $O\left(x^{-N}\right)$ and do not contribute to the $|x| \rightarrow \infty$ contours. Conversely, if $f$ is an analytic function, no such finite series representation is immediately apparent. 
By rescaling and translation any polynomial of order $N$ can be brought to form $f(x)=x^{N}-c x^{N-2}-\ldots$ Hence the last term is given by

$$
\oint \frac{d x}{2 \pi i} \frac{1}{x} \frac{f^{\prime}(x)^{k}}{(f(x) / x)^{k}}=\oint \frac{d x}{2 \pi i} \frac{1}{x} \frac{N^{k} x^{k(N-1)}\left(1+O\left(x^{-2}\right)\right)}{x^{k(N-1)}\left(1+O\left(x^{-2}\right)\right)}=N^{k} .
$$

The first term in (9) is a sum over the zeros of $f(x)$

$$
C(k):=\oint \frac{d x}{2 \pi i} \frac{f^{\prime}(x)^{k}}{f(x)}=\sum_{f(x)=0} f^{\prime}(x)^{k-1} .
$$

By the chain rule (3), the $n$th iterate sum is given by

$$
C_{n}(k)=\sum_{f^{n}(x)=0} f^{n^{\prime}}(x)^{k-1}=\sum_{f^{n-1}(y)=0} f^{(n-1)^{\prime}}(y)^{k-1} \sum_{f(x)=y} f^{\prime}(x)^{k-1} .(12
$$

For $k \leq 3$, the sum over zeros of $f(x)=0$ can be replaced [7] by the pre-images $f(x)=y$ of arbitrary constant $y$, as for $k=2,3$ the difference

$$
\begin{aligned}
& \oint \frac{d x}{2 \pi i}\left\{\frac{f^{\prime}(x)^{k}}{f(x)-y}-\frac{f^{\prime}(x)^{k}}{f(x)}\right\} \\
& =\sum_{j=2}^{k} y^{j-1} \oint \frac{d x}{2 \pi i} \frac{\left(N x^{(N-1)}-(N-2) c x^{N-3}-\ldots\right)^{k}}{\left(x^{N}-c x^{N-2}-\ldots\right)^{j}}
\end{aligned}
$$

vanishes, and hence by induction the $n$th iterate sum (12) is given by [7]

$$
C_{n}(2)=C(2)^{n} \quad C_{n}(3)=C(3)^{n} .
$$

The value of the sum $C(k)$ depends on the parametrization of the particular polynomial. For example, for the cubic polynomial

$$
f(x)=x^{3}-c x-d
$$

we have

$$
C(2)=\oint \frac{d x}{2 \pi i} \frac{\left(3 x^{2}-c\right)^{2}}{x^{3}-c x-d}=\oint \frac{d x}{2 \pi i} 3^{2} x\left(1-\frac{2 c}{3 x^{2}}+\frac{c}{x^{2}}+\ldots\right)=3 c .
$$

If the polynomial is even, $f(x)=g\left(x^{2}\right)$, the terms for $(x / f(x))^{j}$ for which $k+j$ is odd vanish, as

$$
\oint \frac{d x}{2 \pi i} \frac{f^{\prime}(x)^{k}}{x}\left(\frac{x}{f(x)}\right)^{j}=2^{k} \oint \frac{d x x^{k+j-1}}{2 \pi i} \frac{g^{\prime}\left(x^{2}\right)^{k}}{g\left(x^{2}\right)^{j}} .
$$

For example, for even polynomials $C(2)=0$. For general $k$ there are no formulas of the above type for the other terms in the expansion (9).

\subsection{Sum rules for periodic orbits of any polynomial}

Our periodic orbits sum rules are obtained by pushing the contour to $|x| \rightarrow \infty$ in (8). The first periodic orbits sum rule for 1- $d$ maps follows from

$$
T(0)=\oint \frac{d x}{2 \pi i} \frac{1}{f(x)-x}=\oint \frac{d x}{2 \pi i} \frac{1}{x^{N}\left(1+O\left(x^{-1}\right)\right)}=0,
$$


hence

$$
\sum_{i \in \operatorname{Fix}\left(f^{n}\right)} \frac{1}{\Lambda_{i}-1}=0
$$

The second and third periodic orbits sum rules follow from (10):

$$
\begin{aligned}
& T_{n}(1)=\oint \frac{d x}{2 \pi i} \frac{f^{n^{\prime}}(x)}{f^{n}(x)-x}=\sum_{i \in \operatorname{Fix}\left(f^{n}\right)} \frac{\Lambda_{i}}{\Lambda_{i}-1}=N^{n} \\
& T_{n}(2)=\oint \frac{d x}{2 \pi i} \frac{f^{n \prime}(x)^{2}}{f^{n}(x)-x}=\sum_{i \in \operatorname{Fix}\left(f^{n}\right)} \frac{\Lambda_{i}^{2}}{\Lambda_{i}-1}=N^{2 n}+C(2)^{n} .
\end{aligned}
$$

The corresponding Fredholm determinants follow from (6):

$$
\begin{aligned}
& F(z, 0)=1, \quad F(z, 1)=1-z N, \\
& F(z, 2)=\left(1-z N^{2}\right)(1-z C(2)) .
\end{aligned}
$$

The $T_{n}(0), T_{n}(1)$ sum rules will be generalized to $d$-dimensions in section 6. Using (9) we obtain the fourth sum rule

$$
T_{n}(3)=C(3)^{n}+\oint \frac{d x}{2 \pi i} \frac{x f^{n \prime}(x)^{3}}{f^{n}(x)^{2}}+N^{3 n}=\sum_{i \in \operatorname{Fix}(f)} \frac{\Lambda_{i}^{3}}{\Lambda_{i}-1} .
$$

For even polynomials the second term vanishes.

These are exceedingly simple sum rules and expressions for Fredholm determinants, and we can already discern both their utility and the ways in which they might fall short of what we need in order to perform efficient dynamical systems computations. By taking into account all periodic points, not just the real ones, these sum rules dispense with the infinite intricacies of controlling the parameter dependence of symbolic dynamics of systems as simple as a parabola. However, there are clearly very important physical effects that our sum rules are blind to: the most striking is the role played by bifurcations. While at a bifurcation real dynamics changes qualitatively, going from hyperbolic through intermittent to a stable attractor, the corresponding complexified "signed" sum rules are arranged precisely in such a way that these effects cancel.

Starting with $T(3)$, the sum rules cannot be cast in form applicable to arbitrary polynomials, so we now specialize to the quadratic polynomials. We refer the reader interested in the transfer operators weighted by more general rational polynomials to ref. [8].

\section{Sum rules for the quadratic map}

The quadratic polynomial

$$
f(x)=x^{2}-c
$$


is the simplest example of a non-linear 1- $d$ mapping (note the unconventional sign of the parameter $c$, chosen to simplify our final formulas). By the chain rule (3) for the derivative of an iterated function, the stability of an n-cycle $\left\{x_{1}, x_{2}, \cdots, x_{n}\right\}$ is given by

$$
\Lambda=2^{n} x_{1} x_{2} \cdots x_{n} .
$$

We shall now derive an explicit formula for the sum rules and Fredholm determinants for transfer operators (2) weighted by integer powers of the unstable eigenvalue $\Lambda^{k}$ in (5). The derivation is based on the following simple observation which relates contour integrals of functions of the $n$th iterate $f^{n}(x)$ to contour integrals of functions of $f^{n+1}(x)$ : for a quadratic polynomial and a sufficiently large contour, a change of the integration variable $x \rightarrow y=f(x)$ wraps the contour around twice

$$
\oint_{\gamma} \frac{d x f^{\prime}(x)}{2 \pi i} h(f(x))=2 \oint_{\gamma} \frac{d y}{2 \pi i} h(y) .
$$

The factor 2 arises because for large $|x|$ the phase of $f(x) \approx x^{2}$ advances at twice the speed of the phase of $x$, see figure 1. Reconsider now the sum (9) with the last term evaluated as in (10):

$$
T_{n}(k)=\oint \frac{d x}{2 \pi i} \frac{f^{n^{\prime}}(x)^{k}}{x} \sum_{s=1}^{k-1}\left(\frac{x}{f^{n}(x)}\right)^{s}+2^{k n} .
$$

The individual terms in the sum are the diagonal terms of the $[(k-1) \times(k-1)]$ matrix

$$
\mathbf{A}_{r s}^{(n)}:=\oint \frac{d x}{2 \pi i} \frac{x^{s-1}}{f^{n}(x)^{r}} f^{n^{\prime}}(x)^{k}, \quad r, s=1,2, \cdots, k-1,
$$

so the $n$th iterate trace can be expressed as

$$
T_{n}(k)=\operatorname{tr} \mathbf{A}^{(n)}+2^{k n} .
$$

Trivially, for even polynomials $A_{r s} \neq 0$ if $k$ and $s-1$ are either both odd or both even. The motivation for introducing the matrix $\mathbf{A}^{(n)}$ is the observation that its elements are a convenient basis for relating the successive trace sums $T_{n}(k) \rightarrow T_{n+1}(k)$. By (16)

$$
\mathbf{A}_{r s}^{(n+1)}=\oint \frac{d x f^{\prime}(x)}{2 \pi i} \frac{x^{s-1} f^{\prime}(x)^{k-1}}{f^{n}(f(x))^{r}} f^{n^{\prime}}(f(x))^{k}
$$

vanishes for even polynomial $f$ unless $s+k=$ even. Hence we can substitute $x^{s-1} f^{\prime}(x)^{k-1}=2^{k-1} x^{s+k-2}=2^{k-1}(y+c)^{\frac{s+k-2}{2}}$, and applying (24) obtain

$$
\mathbf{A}_{r s}^{(n+1)}=2^{k} \oint \frac{d y}{2 \pi i} \frac{(y+c)^{\frac{k+s-2}{2}}}{f^{n}(y)^{r}} f^{n^{\prime}}(y)^{k} .
$$

We note that this step relies on the very simple form of the quadratic polynomial, so a generalization to arbitrary polynomial mappings is not immediate. Now expand binomially the $(y+c)$ term

$$
\mathbf{A}_{r s}^{(n+1)}=2^{k} \sum_{p=0}^{\frac{k+s-2}{2}}\left(\begin{array}{c}
\frac{k+s-2}{2} \\
p
\end{array}\right) c^{\frac{k+s-2}{2}-p} \mathbf{A}_{r, p+1}^{(n)}
$$


and observe that the successive $\mathbf{A}^{(n)}$ are obtained by multiplication by the $[(k-1) \times$ $(k-1)$ ] dimensional matrix

$$
\mathbf{L}_{(k) r s}:=\left\{\begin{array}{cl}
0 & \text { if } k, s \text { differ in parity } \\
\left(\begin{array}{c}
\frac{k+s-2}{2} \\
r-1
\end{array}\right) c^{\frac{k+s}{2}-r} & \text { otherwise. }
\end{array}\right.
$$

As $\mathbf{A}^{(0)}=\mathbf{1}$, the $n$th level trace sums are given by the trace of $\mathbf{A}^{(n)}=2^{k n} \mathbf{L}_{(k)}^{n}$, and the Fredholm determinant (6) of the transfer operator (2) is given by

$$
F\left(z / 2^{k}, k\right)=(1-z) \operatorname{det}\left(1-z \mathbf{L}_{(k)}\right)
$$

The first 10 such determinants are tabulated in table 1, and an example is given in Appendix A.5. This is the main result of this section: we have obtained an explicit formula for the spectrum of the transfer operator $\mathcal{L}_{(k)}$ for any positive integer $k$ in terms of the eigenvalues of a finite matrix $\mathbf{L}_{(k)}$. As by the symmetry of $f$ the entries in the half of the columns of (26) vanish, in computations it is convenient to distinguish the odd and even cases, and take $\mathbf{L}_{(k)}$ to be a $[l \times l]$ matrix, $i, j=0,1,2, \cdots, l-1$ :

$$
\mathbf{L}_{(k) i j}:= \begin{cases}\left(\begin{array}{c}
l+j \\
2 i
\end{array}\right) c^{l+j-2 i} & \text { odd } k=2 l+1 \\
\left(\begin{array}{c}
l+j+1 \\
2 i+1
\end{array}\right) c^{l+j-2 i} & \text { even } k=2 l+2 .\end{cases}
$$

These finite matrices were first introduced by Levin, Sodin, and Yuditskii, section 4 of ref. [8].

Table 1. Fredholm determinants (27) for the quadratic map transfer operators $\mathcal{L}_{(k)}$, for $k$ a positive integer.

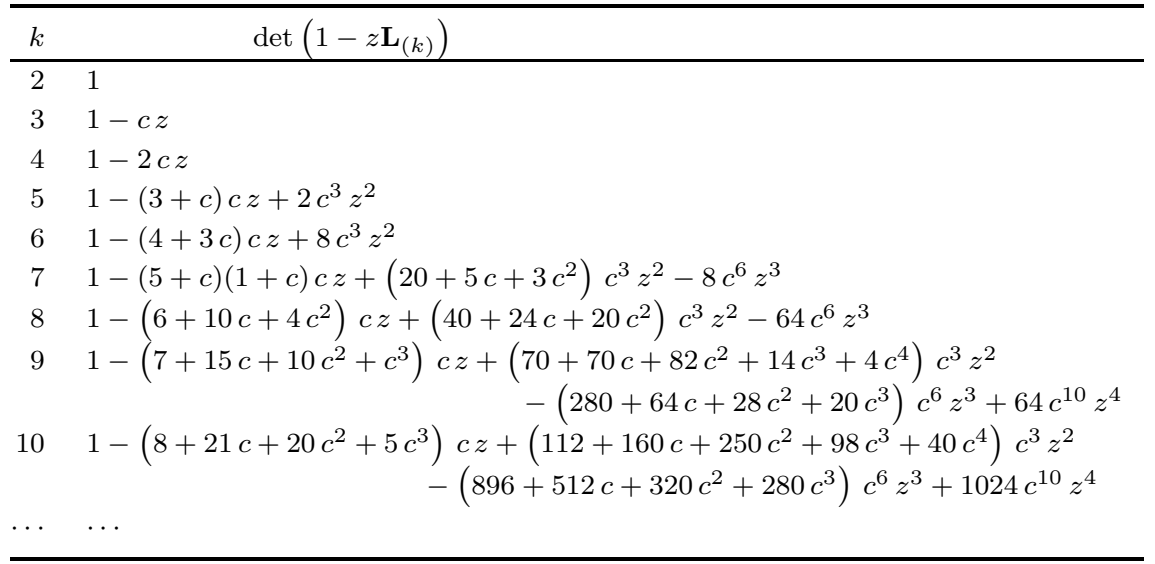




\subsection{Dynamical zeta functions}

Using a different approach, Hatjispiros and Vivaldi [10] have introduced a family of dynamical zeta functions for complex quadratic polynomials, and conjectured that these zeta functions are of a particularly simple rational form. We note that the formula (27) proves the conjectured rationality of this family of dynamical zeta functions. However, their method of evaluating the determinants is still of considerable interest, as it appears to be more efficient for evaluation of $\operatorname{det}\left(1-z \mathbf{L}_{(k)}\right)$ for high $k$ than the direct evaluation undertaken here.

For purposes of comparison with ref. 10] we need to relate dynamical zeta functions to the Fredholm determinants given above. For $|\Lambda|>1$, we expand the weight in (5)

$$
\frac{1}{\Lambda^{r}\left(1-1 / \Lambda^{r}\right)}=\frac{1}{\Lambda^{r}} \sum_{j=0}^{\infty} \Lambda^{-j r}
$$

and obtain the product representation of the Fredholm determinant for 1- $d$ maps

$$
F(z, k)=\exp \left(-\sum_{p} \sum_{r=1}^{\infty} \frac{z^{n_{p} r}}{r} \frac{1}{\Lambda_{p}^{r k}\left(\Lambda_{p}^{r}-1\right)}\right)=\prod_{p} \prod_{j=0}^{\infty}\left(1-\frac{z^{n_{p}}}{\Lambda_{p}^{k+j+1}}\right),
$$

where the $p$ product goes over all prime (not self-retracing) cycles. The dynamical zeta function is defined [1] as

$$
1 / \zeta_{(k)}=\prod_{p}\left(1-\frac{z^{n_{p}}}{\Lambda_{p}^{k+1}}\right)
$$

so for 1- $d$ mappings it can be expressed as a ratio of two Fredholm determinants

$$
1 / \zeta_{(k)}=F(z, k) / F(z, k+1) .
$$

The zeta functions of ref. 10 are indexed and normalized differently; here we follow the conventions used in ref. [12]. Our Fredholm determinants are related to the polynomials $G$ of ref. [10] by $\operatorname{det}\left(1-z \mathbf{L}_{(k)}\right)=G_{k-1}(z / 2,-c)$, and our explicit formula (27) is a proof of their conjectures.

\section{Julia set escape rate}

So far we have obtained explicit finite expressions for the spectral determinants of signed transfer operators $\mathcal{L}_{(k)}$ with weight $\left(f^{\prime}\right)^{k}, k \geq 0$. These results can easily be generalized to arbitrary polynomial weights . However, the methods outlined in section 3 fail for weights $\left(f^{\prime}\right)^{k}$ with $k<0$. Especially the case $k=-1$ is physically interesting, because the largest eigenvalue of the operator gives the escape rate $\gamma$ from a given enclosure $\Gamma$, which is defined by the fraction of initial points that stay in $\Gamma$ after $n$ iterations 13

$$
e^{-n \gamma} \approx \frac{\int_{\Gamma} d x d y \delta\left(x-f^{n}(y)\right)}{\int_{\Gamma} d x} .
$$


The signed version of this transfer operator is defined with the weight $\frac{1}{f^{\prime}(y)^{2}}$ and by summing over all real and complex pre-images of $x$ :

$$
\left(\mathcal{L}_{(-1)} \phi\right)(x)=\sum_{y: f(y)=x} \frac{\phi(y)}{f^{\prime}(y)^{2}}, \quad x, y \in \mathbf{C} .
$$

If the Julia set is real the two operators coincide.

The spectral determinant of (32) has been computed in ref. [8] for quadratic maps $f(x)=x^{2}-c$. If $c$ lies outside the Mandelbrot set $M$, the critical point is attracted by infinity and the spectral determinant is given by the entire function

$$
F(z,-1)=1+\sum_{n=1}^{\infty} \frac{z^{n}}{2^{n} f(0) f^{2}(0) \cdots f^{n}(0)},
$$

which involves only forward iterates of the critical point of $f$. If $c$ lies inside the Mandelbrot set, the critical point is attracted by a limit cycle. If the stability of this cycle is different from zero, (33) is the form of the spectral determinant for small $z$ and can be meromorphically continued. If on the other hand the critical point is periodic with period $n_{p}$, the spectral determinant is a polynomial [8].

In this section first we outline the derivation of this result (for details consult refs. [8]). Then we generalize it and show that it is correct also for a dense set of maps on the boundary of the Mandelbrot set.

The Julia set $J$ is the closure of all repelling periodic orbits. If $c$ lies outside the Mandelbrot set $M$, the Julia set resembles the Cantor dust. To prove (33) we have to use analyticity properties of the transfer operator and its adjoint. One starts by defining the evolution operator $\mathcal{L}_{(-1)}$ on the space of functions $\psi$ which are locally analytic in a small neighbourhood around $J$. Then one can prove that the spectrum of $\mathcal{L}_{(-1)}$ is a point spectrum with the only possible condensation at zero. The dual or adjoint operator $\mathcal{L}_{(-1)}^{\star}$ of the transfer operator $\mathcal{L}_{(-1)}$, defined on densities $\phi^{\star}$ dual to the functions $\psi$, then has the same spectrum. The duality is with respect to the bilinear form

$$
\phi^{\star}(\psi)=\oint_{\gamma} \frac{d x}{2 \pi i} \phi(x) \psi(x), \text { and } \phi(x)=\phi^{\star}\left(\frac{1}{x-.}\right)
$$

which is natural if one deals with analytic functions. The function $\phi(x)$ is holomorphic on the complement of $J$ and vanishes at infinity. The contour $\gamma$ encircles the Julia set and lies in the common domain of analyticity of $\phi$ and $\psi$. The spectral equation $\phi^{\star}-z \mathcal{L}_{(-1)}^{\star} \phi^{\star}=0$ can then be written as

$$
\oint_{\gamma} \frac{d x}{2 \pi i} \psi(x)\left(\phi(x)-z \frac{1}{f^{\prime}(x)} \phi(f(x))\right)=0
$$

for all $\psi$. Therefore

$$
R(x):=\phi(x)-z \frac{1}{f^{\prime}(x)} \phi(f(x))
$$


has to be holomorphic around $J$ and can have poles only if $\frac{1}{f^{\prime}(x)}$ has poles, because $\phi$ is analytic outside $J$.

Specializing to the map $f(x)=x^{2}-c$, the adjoint equation (35) leads to

$$
R(x)=\phi(x)-\frac{z}{2 x} \phi\left(x^{2}-c\right) .
$$

We conclude that $R$ vanishes at infinity because $\phi$ does. Furthermore $R$ can only have a pole of order one at zero, the unique critical point $x_{c}$ of $f$. That means we have either $R(x)=\frac{1}{x}$ (up to a multiplicative constant) or $R(x)=0 . \quad R(x)=0$ is contradictory because it yields eigenfunctions equal to zero. By iterating (37) after solving it for $\phi(x)$ we get

$$
\phi(x)=\frac{1}{x}+\sum_{n=1}^{\infty} \frac{z^{n}}{2^{n} f^{n}(x) \prod_{j=0}^{n-1} f^{j}(x)} .
$$

Now note that $\phi$ is only an eigenfunction if it is holomorphic at the poles of $R$, that means at zero in our case $\left(f^{\prime}\left(x_{c}\right)=0\right)$. Therefore

$$
\begin{aligned}
0 & =\operatorname{Res}_{x=0}[\phi(x)] \\
& =1+\sum_{n=1}^{\infty} \frac{z^{n}}{2^{n} f(0) f^{2}(0) \cdots f^{n}(0)}=F(z,-1)
\end{aligned}
$$

is an equation for the characteristic values of $\mathcal{L}_{(-1)}$ meaning that (38) is an eigenfunction if and only if $z$ is an eigenvalue. For a generalization of this method to arbitrary rational maps see [8].

The form (33) for the spectral determinant for the map $f(x)=x^{2}-c$ holds true, if $c$ lies outside or inside the Mandelbrot set $M$ [8]. However, the method fails if $c$ lies on the border of $M$, and in this case the form of the spectral determinant is unknown.

As a generalization to [8] we show here that on a dense subset of the border of $M$, the so called Misiurewicz points, formula (33) still applies. The Misiurewicz points are defined by the requirement that the critical point $x_{c}=0$ is preperiodic [17]. In figure 2 we introduce the notation for preperiodic points. If we take the validity of (33) for granted, a straightforward calculation yields that for Misiurewicz points the spectral determinant is in fact a rational function composed of a transient part and a periodic contribution

$$
F(z,-1)=1+\sum_{n=1}^{t-2} \frac{z^{n}}{2^{n} \prod_{j=1}^{n} f^{j}(0)}+\frac{z^{t-1}}{2^{t-1} \prod_{j=1}^{t-1} f^{j}(0)} \frac{\sigma(z)}{\left(1-\frac{z^{n_{p}}}{\Lambda_{p}}\right)} .
$$

Here

$$
\sigma(z)=1+\frac{z}{2 x_{1}}+\frac{z^{2}}{4 x_{1} x_{2}}+\ldots+\frac{z^{n_{p}-1}}{2^{n_{p}-1} x_{1} \cdots x_{n_{p}-1}},
$$

$\Lambda_{p}=2^{n_{p}} x_{1} \cdots x_{n_{p}}$ is the stability of the periodic orbit, $n_{p}$ is the period and $t$ the transient time of the Misiurewicz point. The $n_{p}+t-2$ zeroes of $F(z,-1)$ are the 


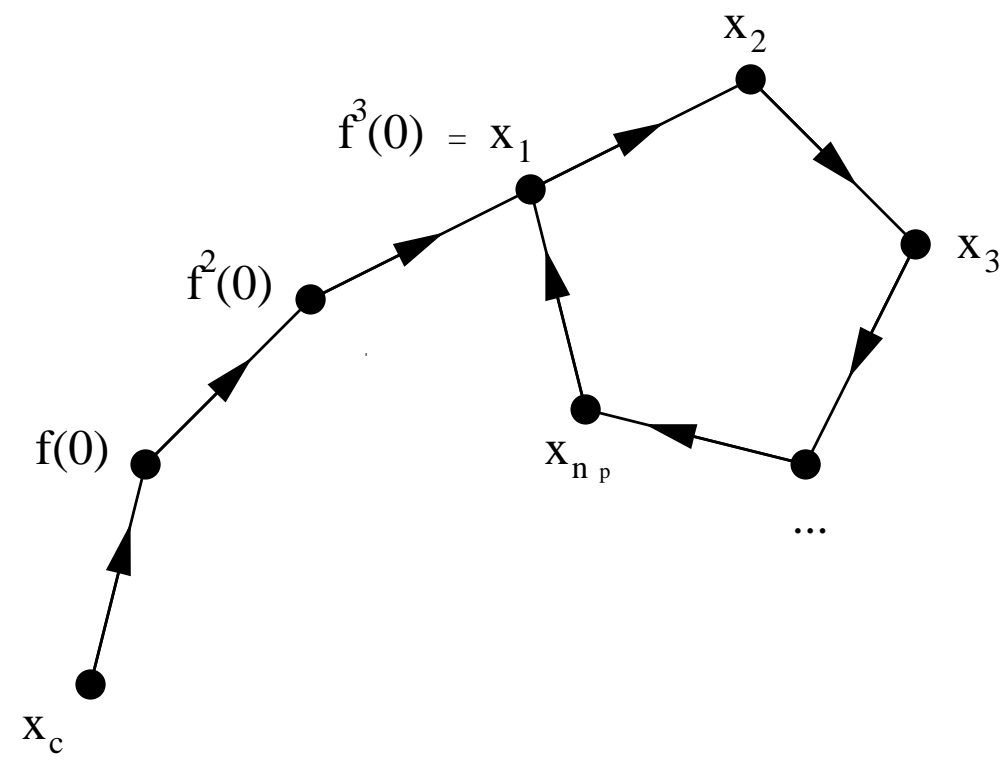

Figure 2. The critical point $x_{c}=0$ is a preperiodic point with transient time $t=3$ and period $n_{p}=5$. In this case the parameter $c$ of the quadratic map is called a Misiurewicz point. Note that the periodic orbit is a repeller.

characteristic values of $\mathcal{L}_{(-1)}$. $F$ has $n_{p}$ poles which lie on a cycle around the origin in the complex $z$-plane. As the period $n_{p}$ of the orbit increases to infinity, these poles built up a wall which limits the region of analyticity.

Presumably, formula (40) can be proved by a generalization of the arguments in [8]. Here we check it using cycle expansion, i.e. the power series representation (6) of the Fredholm determinant [14, 13]. As the first example we compute the spectral determinant for the Ulam map $f(x)=x^{2}-2$. For this map the critical point $x_{c}=0$ iterates via $f(0)=-2$ to the unstable fixed point $x^{\star}=2$. That means we have $t=2$ and $n_{p}=1$, and formula (40) yields

$$
F(z,-1)=1-\frac{z}{4} \frac{1}{1-z / 4}=\frac{1-z / 2}{1-z / 4}
$$

in agreement with the cycle expansion result of ref. [14], where this formula is derived by observing that for all cycles the stability is $\left|\Lambda_{p}\right|=2^{n_{p}}$, with exception of the fixed point $x_{0}$ for which $\Lambda_{0}=4$. However, the result for a general Misiurewicz polynomial is not as trivial.

Next we check (40) by a numerical computation of the spectral determinant via complex cycle expansion. The method is described in detail in [18]. Here we will only state the results. As a first example we study the case $c=1.5436890126920763616 \ldots$, for which the critical point $x_{c}=0$ iterates after three steps into a fixed point $x^{\star}=c(1-c)$, so $t=3$ and $n_{p}=1$. Therefore we expect a spectral determinant 


\begin{tabular}{|c|c|c|}
\hline$n$ & $a_{n}$ & $\frac{a_{n}}{a_{n+1}}$ \\
\hline 0 & 1.00000000000000000 & 3.087378025384153 \\
1 & -0.3238994356305212 & 1.678573510428349 \\
2 & -0.1929611265865065 & -1.678573510428322 \\
3 & 0.1149554222008833 & -1.678573510428341 \\
4 & -0.0684839963735331 & -1.678573510428292 \\
5 & 0.0407989259618778 & -1.678573510428285 \\
6 & -0.0243057129809394 & -1.678573510428318 \\
7 & 0.0144799812638157 & -1.678573510428306 \\
8 & -0.0086263611178524 & \\
\hline
\end{tabular}

Table 2. Spectral determinant for a chaotic quadratic map up to order 8 in complex cycle expansion. For $n>1$, the ratio $a_{n} / a_{n+1}$ of the cycle expansion coefficients (6) computed from $2^{n}$ periodic points equals $\Lambda=2 c(1-c)=-1.6785735104283$ to machine precision, in agreement with $(43)$

of the form

$$
F(z,-1)=1+\frac{z}{2 f(0)}+\frac{z^{2}}{4 f(0) f^{2}(0)} \frac{1}{\left(1-\frac{z}{2 x^{\star}}\right)}
$$

with $f(0)=-c$ and $f^{2}(0)=-x^{\star}=c(c-1)$. In table 2 the coefficients $a_{n}$ of the cycle expansion for the spectral determinant $F(z,-1)=\sum_{n=0}^{\infty} a_{n} z^{n}$ are depicted. The second column of the table demonstrates that they indeed decrease geometrically with the correct ratio. The remaining coefficients can be checked numerically or computed analytically from the traces of $\mathcal{L}_{(-1)}$ and $\mathcal{L}_{(-1)}^{2}$. In either case we arrive at (43). Because the numerator of the spectral determinant is quadratic there are two characteristic values, namely $z=c^{2} *(1-c)=-1.295597742522084$ and $z=2$. That means the spectrum is real and there is a real pole at $z=2 c(1-c)=$ -1.678573510428321 . The escape rate (31) is given by the trivial largest eigenvalue $\gamma=\log (2)$.

As our last example we take the map $f(x)=x^{2}+i$, for which $x_{c}=0$ has the trajectory

$$
0 \mapsto i \mapsto x_{1}=-1+i \mapsto x_{2}=-i \mapsto-1+i \mapsto \ldots,
$$

so $t=2$ and $n_{p}=2$. The numerical cycle expansion 18 yields

$$
F(z,-1)=\frac{\frac{z}{2 i}-\frac{z^{2}}{4+4 i}}{1-\frac{z^{2}}{4+4 i}},
$$

where by 40 we had expected

$$
F(z,-1)=1+\frac{z}{2 f(0)} \frac{1+\frac{z}{2 x_{1}}}{1-\frac{z^{2}}{4 x_{1} x_{2}}} .
$$


After short inspection the two expressions turn out to be the same.

Any number of such examples can be worked out in the same way. In every case a scaling of the numerical coefficients confirms the rational form of the Fredholm determinant for the Misiurewicz polynomials.

\section{Multi-dimensional polynomial mappings}

In the multi-dimensional case a typical contour integral representation of a periodic orbit sum is of form

$$
T(0)=\oint \frac{d x_{1}}{2 \pi i} \oint \frac{d x_{2}}{2 \pi i} \cdots \oint \frac{d x_{d}}{2 \pi i} \prod_{\alpha=1}^{d} \frac{1}{f_{\alpha}(x)-x_{\alpha}} .
$$

For example, in 2 dimensions the contour integral picks up a contribution from each fixed point $i$

$$
f_{1}\left(x_{i}, y_{i}\right)-x_{i}=0, \quad f_{2}\left(x_{i}, y_{i}\right)-y_{i}=0 .
$$

The integral can be converted into a sum of local contour integrals around linearized neighbourhoods of the fixed points $\left(x_{i}, y_{i}\right)$

$$
T(0)=\sum_{i} \oint \frac{d z_{i 1}}{2 \pi i} \oint \frac{d z_{i 2}}{2 \pi i} \frac{1}{\left(z_{i 1}-J_{11} z_{i 1}-J_{12} z_{i 2}\right)\left(z_{i 2}-J_{21} z_{i 1}-J_{22} z_{i 2}\right)},
$$

where $x=x_{i}+z_{i 1}, y=y_{i}+z_{i 2}$, and $\mathbf{J}_{i}$ is the Jacobian matrix

$$
\left[\begin{array}{ll}
J_{11} & J_{12} \\
J_{21} & J_{22}
\end{array}\right]=\left[\begin{array}{ll}
\partial_{x} f_{1}(x, y) & \partial_{y} f_{1}(x, y) \\
\partial_{x} f_{2}(x, y) & \partial_{y} f_{2}(x, y)
\end{array}\right]_{(x, y)=\left(x_{i}, y_{i}\right)} .
$$

Completing the integrals we get for 2 dimensions

$$
T(0)=\sum_{i \in \operatorname{Fix}(f)} \frac{1}{\left(1-J_{11}\right)\left(1-J_{22}\right)-J_{12} J_{21}},
$$

and in $d$ dimensions

$$
T(0)=\sum_{i \in \operatorname{Fix}(f)} \frac{1}{\operatorname{det}\left(1-\mathbf{J}_{i}\right)}=0
$$

where $\mathbf{J}_{i}$ is the Jacobian (monodromy matrix) of the $i$ th fixed point. Pushing all contours in (46) off to infinity yields the sum rule $T(0)=0$. Just as in the 1dimensional case (17), $T(0)$ vanishes when all fixed points, real and complex, are included in the sum.

\subsection{A sum rule related to the fast kinematic dynamo}

Our second multi-dimensional sum rule arises in the study of a 2- $d$ Poincaré map of a model of the fast kinematic dynamo [6]. The fast kinematic dynamo is a problem of 
passive vector field advection: the dynamo rate is determined by the overall growth of a small vector field embedded into the flow. In this case the transfer operator (2) is weighted by the Jacobian matrix $\mathbf{J}^{t}(x, y)$, a multiplicative function evaluated along the trajectory with initial point $(x, y)$. For 2- $d$ maps this transfer operator leads to traces of form $\operatorname{tr} \mathcal{L}=\sum \operatorname{tr} \mathbf{J} /|\operatorname{det}(1-\mathbf{J})|$. We can derive a sum rule for the corresponding signed measure trace sum:

$$
\begin{aligned}
T(1) & =\oint \frac{d x}{2 \pi i} \oint \frac{d y}{2 \pi i} \frac{\operatorname{tr} \mathbf{J}(x, y)}{(x-f(x, y))(y-g(x, y))} \\
& =\sum_{i \in \operatorname{Fix}(f)} \frac{\operatorname{tr} \mathbf{J}_{i}}{\operatorname{det}\left(1-\mathbf{J}_{i}\right)} .
\end{aligned}
$$

The 2- $d$ matrix identity

$$
\begin{aligned}
\frac{\operatorname{tr} \mathbf{J}}{\operatorname{det}(1-\mathbf{J})} & =\frac{\operatorname{tr} \mathbf{J}}{1-\operatorname{tr} \mathbf{J}+\operatorname{det} \mathbf{J}} \\
& =\frac{1}{\operatorname{det}(1-\mathbf{J})}-\frac{1}{\operatorname{det}\left(1-\mathbf{J}^{-1}\right)}-1
\end{aligned}
$$

yields our sum rule

$$
T(1)=\sum_{i \in \operatorname{Fix}(f)} \frac{\operatorname{tr} \mathbf{J}_{i}}{\operatorname{det}\left(1-\mathbf{J}_{i}\right)}=-N,
$$

where we have used the fact that $T(0)$ vanishes for both the forward and the time reversed flow. As in the 1- $d$ case (18), $T(1)$ counts the number of the fixed points of the map; in polynomial maps this is given by the order of the polynomial.

\subsection{An application: numerical checks of cycle sums}

As an example for the utility of sum rules for higher dimensional polynomial mappings, consider the 2-dimensional Hénon map

$$
x_{k+1}=1-a x_{k}^{2}+y_{k}, \quad y_{k+1}=b x_{k} .
$$

For the complete repeller case (all binary sequences are realized), the Hénon map is a realization of the complete Smale horseshoe. Cycle stabilities are easily computed numerically [12. We have verified numerically the sum rules (47) and (48) for a volume preserving Hénon map repeller $(b=-1)$ by substituting the cycle stabilities of cycles up to length 12 into

$$
\begin{gathered}
\sum_{i \in \operatorname{Fix}\left(f^{n}\right)} \frac{1}{\Lambda_{i}\left(1-1 / \Lambda_{i}\right)^{2}}=0 \\
\sum_{i \in \operatorname{Fix}\left(f^{n}\right)} \frac{\Lambda_{i}+1 / \Lambda_{i}}{\Lambda_{i}\left(1-1 / \Lambda_{i}\right)^{2}}=2^{n}
\end{gathered}
$$


(here $\Lambda_{i}$ is the expanding eigenvalue of $\mathbf{J}_{i}$ ). The first sum can be used to check the accuracy of the periodic orbit data used to compute the escape rate $\gamma$ of the Hénon repeller

$$
\sum_{i \in \operatorname{Fix}\left(f^{n}\right)} \frac{1}{\left|\Lambda_{i}\right|\left(1-1 / \Lambda_{i}\right)^{2}}=e^{-n \gamma}+\text { (non-leading eigenvalues). }
$$

If we add (50) to the above sum, for complete horseshoe all inverse hyperbolic cycle contributions with $\Lambda_{i}<0$ cancel, and hence only a half of the cycles suffices to to compute the escape rate. The second sum rule can be used to check the accuracy of the data used to estimate the kinematic dynamo rate $\eta$

$$
\sum_{i \in \operatorname{Fix}\left(f^{n}\right)} \frac{\Lambda_{i}+1 / \Lambda_{i}}{\left|\Lambda_{i}\right|\left(1-1 / \Lambda_{i}\right)^{2}}=e^{n \eta}+\text { (non-leading eigenvalues). }
$$

It was a numerical discovery of these sum rules computed in connection with the kinematic dynamo model of ref. [6] that lead us to the contour integral formulation and derivation of all other sum rules given in this paper.

\section{Farey map (or spin-chain) thermodynamics}

So far, our examples were based on the contour integral technique. In this section we shall use a very different, number theoretic approach to compute the spectrum of transfer operators. We shall consider the Farey map, figure 3, a combination of two Moebius transformations

$$
f(x)=\left\{\begin{array}{lll}
x /(1-x) & x \in[0,1 / 2) \\
(1-x) / x & x \in[1 / 2,1]
\end{array} .\right.
$$

The Farey map arises in a variety of contexts: dynamics of circle maps, dynamical renormalization theory, statistical mechanics, number theory. For dynamical systems motivation we refer the reader to the review 19. In particular, the Farey map plays the same role vis-a-vis the shift circle map that the highly nontrivial circle map presentation function 20, 21 plays in the golden-mean renormalization theory, and provides a controllable setting to test some of the ideas that arise in that context. Another motivation comes from statistical mechanics, where Farey model thermodynamics corresponds to a number-theoretical model of an infinite ferromagnetic spin chain with effective $r^{-\alpha}$ interaction [11], with phase transition of Thouless type at $\alpha=2$. For the purpose at hand the Farey map is of interest to us because

(i) again an infinity of sum rules can be obtained, but this time without the contour integration approach employed above

(ii) the sum rules apply to the positive measure $|\Lambda|^{\tau}$, in contradistinction to the signed measures of the previous examples 


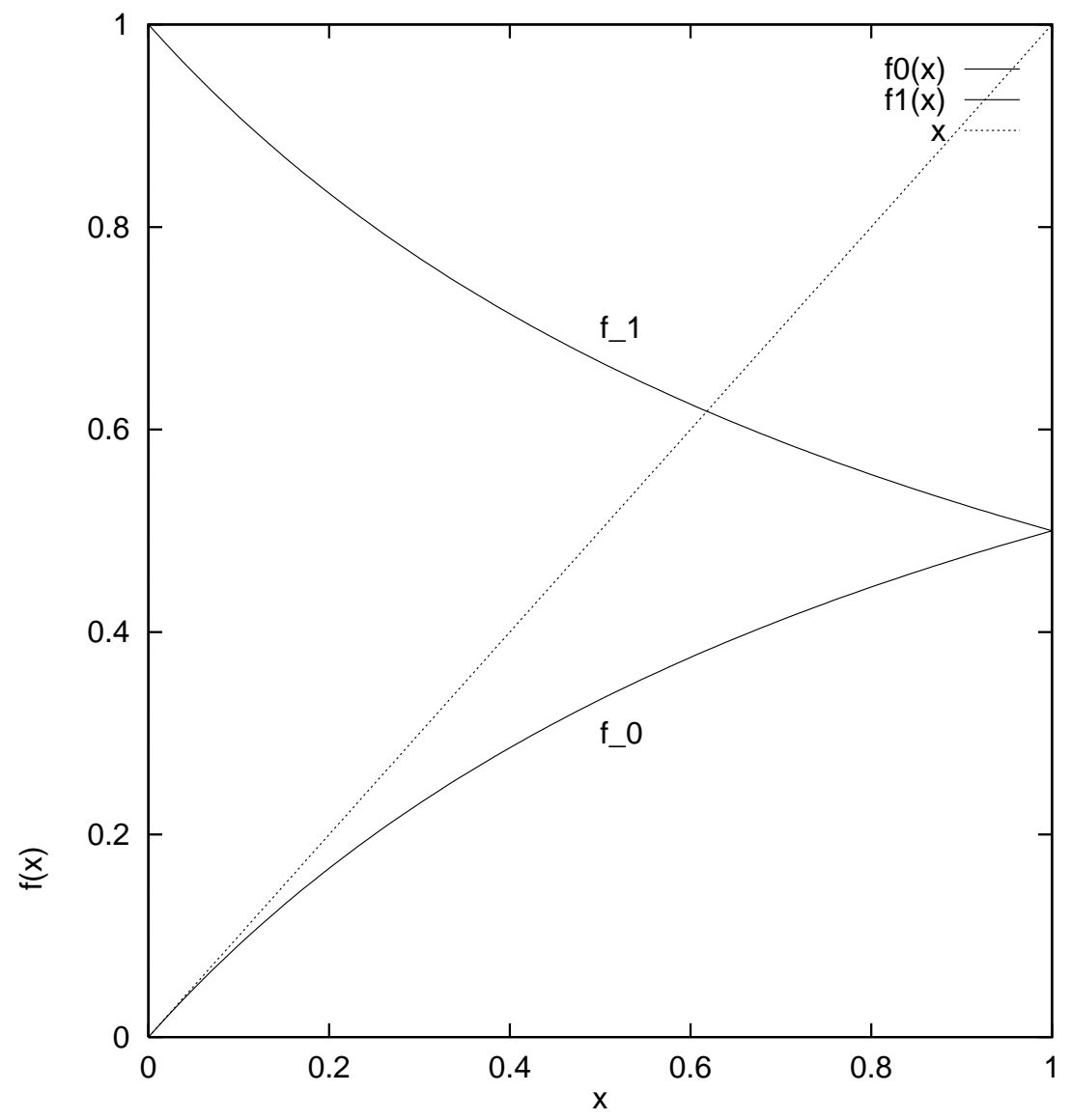

Figure 3. The two branches of the inverse to the Farey map (52).

(iii) the theory can be extended to no-integer exponents $\tau$, with infinite range recursion relations.

Apply the "natural measure" modification of (2)

$$
\mathcal{L}_{(\tau)}(y, x)=\left|f^{\prime}(x)\right|^{\tau} \delta\left(x-f^{-1}(y)\right)
$$

to the Farey map (we have redefined the exponent $(k+1) \rightarrow \tau$ to conform with the notation conventions of refs. [22, 23]), and consider the sum

$$
Z_{n}(\tau):=\int d x\left|f^{n \prime}(x)\right|^{\tau} \delta\left(x-f^{-(n+1)}(1)\right) .
$$

The leading $\mathcal{L}$ eigenvalue $2^{q(\tau)}$ (in the notation of ref. 22]) dominates this sum in the $n \rightarrow \infty$ limit and defines a "thermodynamic" function $q=q(\tau)$. For the Farey map (see figure 3) the pre-images of 1 are

$$
\begin{aligned}
& f(1 / 2)=1, \quad f^{2}(1 / 3)=f^{2}(2 / 3)=1 \\
& f^{3}(1 / 4)=f^{3}(2 / 5)=\cdots=1
\end{aligned}
$$




$$
f^{n}\left(P_{i} / Q_{i}\right)=\cdots=1 .
$$

These fractions form the levels of the Farey tree [24, 25, 26, 27, 23, 28], a number theoretical construction based on the observation that somewhere midway between two small denominator fractions (such as $1 / 2$ and $1 / 3$ ) there is the next smallest denominator fraction (such as $2 / 5)$, given by the "Farey mediant" $\left(P+P^{\prime}\right) /\left(Q+Q^{\prime}\right)$ of the parent mode-lockings $P / Q$ and $P^{\prime} / Q^{\prime}$. The Farey tree is obtained by starting with the ends of the unit interval written as $0 / 1$ and $1 / 1$, and then recursively bisecting intervals by means of Farey mediants. This generates Farey level sets $\mathcal{F}_{n}$ with $2^{n}$ mode-locking widths on each level, figure 4 . In the present context, the $n$th level of

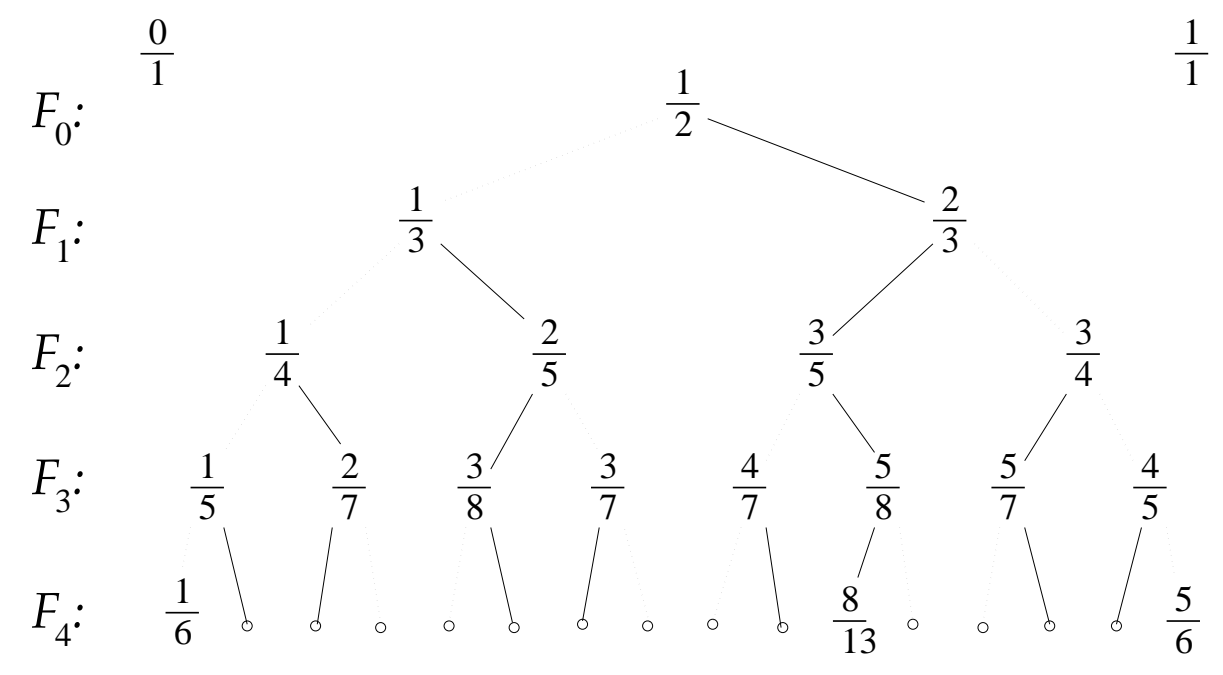

Figure 4. The levels $\mathcal{F}_{n}$ of the Farey tree are generated by the Farey mediant addition rule $P / Q, P^{\prime} / Q^{\prime} \rightarrow\left(P+P^{\prime}\right) /\left(Q+Q^{\prime}\right)$.

the Farey tree is the set of the $2^{n}$ distinct backward iterates $f^{-n}\left(x_{i}\right)=1$ of the Farey map. Furthermore, noting that $f_{o}^{\prime}(x)=1 /(1-x)^{2}, f_{1}^{\prime}(x)=-1 / x^{2}$, it is easily checked that if $P_{i} / Q_{i} \in \mathcal{F}_{n}$, then

$$
\left|f^{n^{\prime}}\left(P_{i} / Q_{i}\right)\right|=Q_{i}^{2} .
$$

For example

$$
f^{2^{\prime}}(1 / 3)=f^{\prime}(1 / 3) f^{\prime}(1 / 2)=(1-1 / 3)^{-2}(1-1 / 2)^{-2}=3^{2}
$$

Hence the sum (54) is the sum over denominators of the Farey rationals of the (n-1)th Farey level:

$$
Z_{n}(\tau)=\sum_{i \in \mathcal{F}_{n}} Q_{i}^{2 \tau},
$$

where $Q_{i}$ is the denominator of the $i$ th Farey rational $P_{i} / Q_{i}$. For example

$$
Z_{2}(1 / 2)=4+5+5+4 .
$$


As we shall now show, $Z_{n}(\tau)$ satisfies an exact sum rule for every non-negative integer $2 \tau$. First one observes that $Z_{n}(0)=2^{n}$. It is also easy to check that 24] $Z_{n}(1 / 2)=\sum_{i} Q_{i}=2 \cdot 3^{n}$. More surprisingly, $Z_{n}(3 / 2)=\sum_{i} Q^{3}=54 \cdot 7^{n-1}$. Such sum rules are consequence of the fact that the denominators on a given level are Farey sums of the denominators on preceding levels. In order to exploit this, the following labelling of the Farey denominators introduced by Knauf [29] is convenient:

The Farey denominators $Q_{\sigma}, \sigma \in\left\{s_{1} s_{2} \cdots s_{n}\right\}, s_{i} \in\{0,1\}$, up to the $n$th level of the Farey tree of figure 1 can be labelled in lexical binary order as illustrated in figure 5 . Farey denominators on the $(n+1)$ th Farey tree level are given by [29]

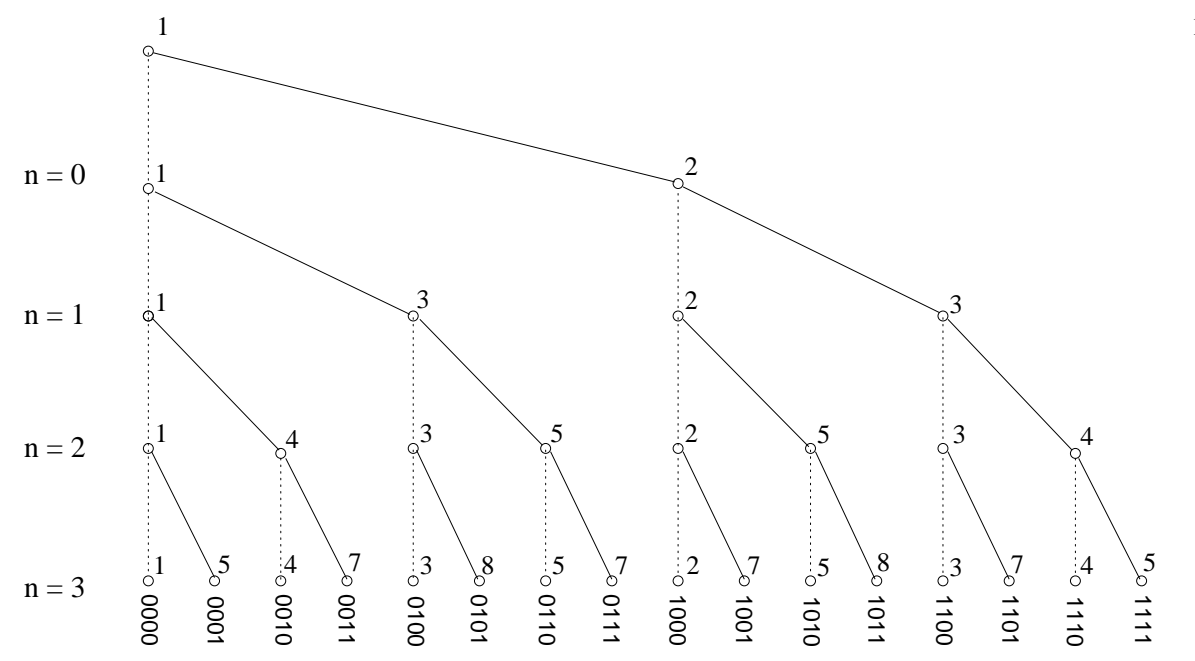

Figure 5. Lexically ordered binary labelling of all Farey denominators up to and including the $n$th Farey tree level, following Knauf 29]. The binary label of the denominator $Q_{\sigma}$ is read off starting with the root of the tree, with $s_{i}=1$ for a full line, and $s_{i}=0$ for a dotted line. For example $Q_{0011}=7, Q_{1000}=2$, etc.. The $2^{n}$ denominators $Q \ldots 1$ preceded by a full line constitute the $n$th Farey level $\mathcal{F}_{n}$.

$Q_{\sigma 0}=Q_{\sigma} \quad$ (carry over all previous Farey denominators)

$Q_{\sigma 1}=Q_{\sigma}+Q_{\bar{\sigma}} \quad$ (compute the $(n+1)$ th Farey tree level),

where $\bar{\sigma}$ is obtained by exchanging 1's and 0's in the string $\sigma, s_{i} \rightarrow 1-s_{i}$, and the recursion is initiated with the empty string values $Q_{.}=Q_{-}=1$. The second rule follows from labelling of nearest neighbours on a given level, see figure 5. By construction, $Q_{\sigma 1}$ is invariant under the $s_{i} \rightarrow 1-s_{i}$ interchange

$$
Q_{\sigma 1}=Q_{\bar{\sigma} 1}
$$

Define a $[2 \tau+1]$-dimensional vector $\phi=\left[\phi_{0}, \phi_{1}, \cdots, \phi_{2 \tau}\right], 2 \tau$ a positive integer, by

$$
\phi_{m}^{(k)}:=\sum_{\sigma \in\left\{s_{1} s_{2} \cdots s_{k}\right\}} Q_{\sigma 1}^{2 \tau-m} Q_{\sigma 0}^{m}=\sum_{i \in \mathcal{F}_{k+1}}\left(\frac{P_{i}}{Q_{i}}\right)^{m} Q_{i}^{2 \tau} .
$$


The zeroth component of $\phi^{(k)}$ is the $k$ th level Farey sum (56)

$$
Z_{k}(\tau)=\phi_{0}^{(k)}=\sum_{\sigma \in\left\{s_{1} s_{2} \cdots s_{k}\right\}} Q_{\sigma 1}^{2 \tau} .
$$

Motivation for constructing the vector $\phi^{(k)}$ is the observation that its remaining entries exhaust all combinations that arise in exponentiating $Q_{\sigma 1}=Q_{\sigma}+Q_{\bar{\sigma}}$ to the integer powers $s \leq 2 \tau$

$$
\begin{aligned}
\phi_{m}^{(k+1)} & =\sum_{\sigma \in\left\{s_{1} s_{2} \cdots s_{k+1}\right\}}\left(Q_{\sigma}+Q_{\bar{\sigma}}\right)^{2 \tau-m} Q_{\sigma}^{m} \\
& =\sum_{\sigma \in\left\{s_{1} s_{2} \cdots s_{k}\right\}}\left(Q_{\sigma 1}+Q_{\bar{\sigma} 0}\right)^{2 \tau-m} Q_{\sigma 1}^{m}+\left(Q_{\sigma 0}+Q_{\bar{\sigma} 1}\right)^{2 \tau-m} Q_{\sigma 0}^{m} \\
& =\sum_{\sigma \in\left\{s_{1} s_{2} \cdots s_{k}\right\}}\left(Q_{\sigma 1}+Q_{\sigma 0}\right)^{2 \tau-m}\left(Q_{\sigma 1}^{m}+Q_{\bar{\sigma} 1}^{m}\right),
\end{aligned}
$$

(we have used $\sum_{\sigma}=\sum_{\bar{\sigma}}$ and the $Q_{\sigma 1}=Q_{\bar{\sigma} 1}$ symmetry), and that they form a linear basis for $\phi^{(k+1)}$ :

$$
\begin{aligned}
\phi_{m}^{(k+1)} & =\sum_{r=0}^{2 \tau-m}\left(\begin{array}{c}
2 \tau-m \\
r
\end{array}\right) \sum_{\sigma \in\left\{s_{1} s_{2} \cdots s_{k}\right\}}\left(Q_{\sigma 1}^{2 \tau-r} Q_{\sigma 0}^{r}+Q_{\sigma 1}^{r} Q_{\sigma 0}^{2 \tau-r}\right) \\
& =\sum_{r=0}^{2 \tau-m}\left\{\left(\begin{array}{c}
2 \tau-m \\
r
\end{array}\right)+\left(\begin{array}{c}
2 \tau-m \\
r-m
\end{array}\right)\right\} \phi_{r}^{(k)} .
\end{aligned}
$$

Hence the family of vectors $\phi^{(1)}, \phi^{(2)}, \cdots$, is generated by multiplication $\phi^{(k+1)}=$ $\mathbf{L}_{(\tau)} \phi^{(k)}$ by the $[(2 \tau+1) \times(2 \tau+1)]$ dimensional transfer matrix

$$
\mathbf{L}_{(2 \tau) m r}=\left(\begin{array}{c}
2 \tau-m \\
r
\end{array}\right)+\left(\begin{array}{c}
2 \tau-m \\
r-m
\end{array}\right)
$$

consisting of two Pascal triangles. For example

$$
\mathbf{L}_{(2)}=\left[\begin{array}{lll}
2 & 4 & 2 \\
1 & 2 & 1 \\
1 & 0 & 1
\end{array}\right] \text {. }
$$

The growth of $\phi^{(k)}$ in the $k \rightarrow \infty$ limit (and in particular, its zeroth component (59), the thermodynamic sum $\left.Z_{k}(\tau)\right)$ is given by $1 / z=2^{q(\tau)}$, the leading zero of the $\mathbf{L}_{(2 \tau)}$ characteristic polynomial

$$
F(z, 2 \tau):=\operatorname{det}\left(1-z \mathbf{L}_{(2 \tau)}\right)=1-\left(2 \tau+1+F_{2 \tau+1}\right) z-\ldots,
$$

see table 3. Here $F_{n}=F_{n-1}+F_{n-2}, F_{0}=0, F_{1}=1$, are the Fibonacci numbers. The largest $Q_{i}$ in the $n$th level sum (56) is $F_{n+2}$, so for the large $\tau$ the leading eigenvalue tends to $\rho^{2 \tau}$. The polynomials of table 3 were first computed by Cvitanović and Kennedy, Appendix B of ref. [23]; here we have followed the more elegant derivation due to Contucci and Knauf [11]. 
Table 3. Spectral determinants for the Farey model transfer operator 60, $2 \tau=0,1,2, \ldots, 11$, together with the leading eigenvalue $2^{q(\tau)}$.

\begin{tabular}{rcr}
\hline $2 \tau$ & $2^{q(\tau)}$ & $F(z, \tau)$ \\
\hline 0 & 2 & $1-2 z$ \\
1 & 3 & $1-3 z$ \\
2 & $(2.13578 \cdots)^{2}$ & $(1+z)\left(1-11 z+2 z^{2}\right)$ \\
3 & 7 & $1-5 z+2 z^{2}$ \\
4 & $(1.81346 \cdots)^{4}$ & $1-7 z$ \\
5 & $(1.75813 \cdots)^{5}$ & $\left(1-14 z-47 z^{2}\right.$ \\
6 & $(1.72342 \cdots)^{6}$ & $1-20 z-161 z^{2}-40 z^{3}+4 z^{4}$ \\
7 & $(1.69991 \cdots)^{7}$ & $1-29 z-485 z^{2}-327 z^{3}$ \\
8 & $(1.68313 \cdots)^{8}$ & $\left(1-44 z-1313 z^{2}-88 z^{3}+4 z^{4}\right)$ \\
9 & $(1.67068 \cdots)^{9}$ & $1-65 z-3653 z^{2}-3843 z^{3}$ \\
10 & $(1.66117 \cdots)^{10}$ & $\left(1-156 z-24882 z^{2}+83828 z^{3}+107529 z^{4}\right.$ \\
11 & $(1.65375 \cdots)^{11}$ & $\left(1-100 z-9601 z^{2}-200 z^{3}+4 z^{4}\right)$ \\
12 & $(1.64784 \cdots)^{12}$ & $\left(1-247 z+63659 z^{2}+797003 z^{3}-127318 z^{4}-988 z^{5}+8 z^{6}\right)$ \\
$\cdots$ & $\cdots$ &
\end{tabular}

The Farey model demonstrates that not only are the methods of this paper competitive with the periodic orbits methods, but they are sometimes superior to them. For the Farey model the periodic orbit expansions are studied in detail in refs. [30, 31], and depend on quadratic irrationals rather than the Farey denominators. While such periodic orbit expansions are analytically intractable, this variant of the finite level sums yields $q(\tau)$ exactly for all $2 \tau$ a non-negative integer. Not only that, but Contucci and Knauf 11 have in this case been also able to analytically continue the $\mathbf{L}_{(\tau)}$ matrices to arbitrary real positive $\tau$.

\section{Some speculations}

So far we have derived a series of exact sum rules for very specific simple dynamical systems. In this section we speculate about possible, but at present still largely unexplored applications of the above methods in more general settings.

\subsection{Normal form approximations to flows}

The Poincaré maps of flows of physical interest are in general not polynomials, but smooth analytic functions. However, in practical applications (such as the long term integrations in celestial mechanics) it is often advantageous to replace a differential equation by a normal form that approximates the return map for a Poincaré section 
of the flow, and replace the numerically demanding integration of the flow by a map iteration. Such applications require a test of the accuracy of the normal form approximation; the above sum rules offer an estimate of the quality of approximation, which, as it depends only on cycle eigenvalues, is coordinatization independent. The idea is simple: were the polynomial representation of the flow exact, its cycles would satisfy the above sum rules exactly. By comparing the sums of cycles of the smooth flow to what would be expected were the flow generated by a polynomial we learn how good a polynomial approximation of given order would be, prior to any actual fitting of the flow by an approximate mapping.

As an example, consider a 3 -dimensional flow $\dot{\mathbf{x}}=\mathbf{F}(\mathbf{x}), \quad \mathbf{x}=\left(x_{1}, x_{2}, x_{3}\right)$. We assume that the flow of interest is recurrent, and that given a convenient Poincaré section coordinatized by coordinate pair $(x, y)$, the flow can be described by a 2 dimensional Poincaré map

$$
\begin{aligned}
& x^{\prime}=f(x, y) \\
& y^{\prime}=g(x, y)
\end{aligned}
$$

together with the "ceiling" function $T(x, y)$ which gives the time of flight to the next section for a trajectory starting at $(x, y)$. In general $f$ and $g$ can be complicated functions, but the essential properties of a continuous flow can be modelled by the Hénon map (49), which we take as a local normal form (up to quadratic terms). In the Hamiltonian case, the normal form is

$$
x_{k+1}=1-a x_{k}^{2}-x_{k-1} .
$$

If this were an exact representation of the flow, it would satisfy exactly the sum rules (50) and (51), hence the amount by which they are violated is an indication of how well the flow is approximated by a quadratic normal form. As we are summing signed rather than absolute values, this is an underestimate of the actual error, but possibly of interest even so. While numerical tests of the rule on a 3-disk billiard cycles data of ref. [12] do suggest that for sufficiently large disk-disk separation (63) is not a bad approximation, we have not yet checked whether the best value of the parameters in the approximation can be determined by minimizing the deviation from the exact sum rules.

\subsection{Spectrum of the period-doubling operator}

The generalized transfer operators (2) have an important application [14, 32, 33, 34] in the period-doubling renormalization theory: if $f$ is given by the period-doubling presentation function [28]

$$
\begin{array}{ll}
f_{0}(x)=\alpha g(x), & g\left(\alpha^{-1}\right) \leq x \leq 1 \\
f_{1}(x)=\alpha x, & \alpha^{-1} \leq x \leq \alpha^{-2},
\end{array}
$$

where

$$
g(x)=\alpha g \circ g(x / \alpha) .
$$


is the universal period-doubling renormalization fixed point function, the leading eigenvalue of $\mathcal{L}_{(2)}(y, x)=\delta\left(y-f^{-1}(x)\right) f^{\prime}(y)$ is the Feigenbaum $\delta$, and the spectrum of $\mathcal{L}_{(2)}$ is given by the trace formula [14, 33]

$$
\operatorname{tr} \mathcal{L}_{(2)}=\sum_{i \in \operatorname{Fix}(f)} \frac{\Lambda_{i}^{2}}{\Lambda_{i}-1},
$$

or the associated Fredholm determinant. Here the summation is restricted to the real fixed points in the unit interval; application of our sum rule (19) would require inclusion of the complex periodic points as well. The crudest approximation to $g(x)$ is a quadratic polynomial. This already yields a reasonable estimate without any actual polynomial fitting; according to (19), for a quadratic polynomial the leading eigenvalue (19) is 4, while the period-doubling leading eigenvalue is the Feigenbaum

$\delta=4.66 \ldots$. If (19) is really applicable in this context, the sum rule would require inclusion of contributions of complex periodic points of the presentation function how well that converges has not yet been checked.

\section{A critical summary}

The conventional periodic orbit theory suffers from one serious limitation; the number of spectral eigenvalues computable is in practice limited by the exponential growth in the number of periodic orbits required. This arises because the periodic orbit theory in its trace formula formulation is essentially a tessellation of the phase space by linearized neighbourhoods of periodic points which ignores the fact that these neighbourhoods are interrelated by the analyticity of the flow. This suggests that the local periodic orbit information should be supplemented by global analyticity constraints. Previous to the work presented here, analyticity has been exploited to improve the convergence of cycle expansions by incorporating the shadowing of long cycles by combinations of shorter ones [35, 14], and to prove that the cycle expansions of Fredholm determinants can converge faster than exponentially with the topological cycle truncation length [36, 37. While sometimes very useful, these results apply only to nice hyperbolic flows with finite symbolic dynamics. Most realistic physical flows are not uniformly hyperbolic, and do not have finite Markov partitions. For this reason general analyticity constraints applicable to any flow would be of great interest. Here another observation is very suggestive; analytic continuation of pairs of orbits through inverse bifurcations 38] improves the semi-classical quantization in a regime where the dynamics is non-hyperbolic, and utility of periodic orbits a priori in doubt. Similarly, leading diffraction effects of wave mechanics can be accounted for by inclusion of complex "creeping" orbits [39] into periodic orbits sums. These examples suggest that for generic flows perhaps both the non-hyperbolicity and lack of finite Markov partitions may be tamed by taking into account the totality of periodic orbits, real and complex. 
With such considerations in mind we have here compared two strategies to compute the spectrum of a given transfer operator:

(i) the conventional periodic orbit theory approach: $\operatorname{tr} \mathcal{L}=$ sum over cycles

(ii) the new recursive approach: relate $Z_{n}=\int d x \mathcal{L}^{n}(0, x)=$ sum over pre-images to previous levels sums.

We have established that sometimes the iterative nature of the dynamics enables us to obtain formulas for transfer operator eigenvalues without computing any periodic orbits.

The sum rules so obtained come as something of a pleasant surprise, especially in the multi-dimensional cases, as cycle expansions typically require evaluation of exponentially many cycles up to a given cycle length, and it is not at all obvious that the cycle weights should be related in any simple way. Prior to these results, other than the purely topological Lefschetz fixed point formulas there existed only one exact general result for periodic orbit sums, the flow density conservation 40, 14], and several sum-rules specific to billiards. These are explored in a forthcoming paper by S.F.Nielsen et al. 41]. From the vantage point of this work, the flow density conservation rule is of limited interest, as it has no dynamical content. Even though our sum rules require determining not only real cycles, but also the complex ones (ie. also those forbidden by the symbolic dynamics), they might be useful in practice. There are several immediate uses for such rules; one is to check for the correctness of the numerically calculated cycle weights up to a given cycle length, and another, speculated on in section 8.1, is to estimate the quality of polynomial approximations to smooth flows.

Applied to higher dimensional dynamical systems the new sum rules might shed light on the role of complex periodic orbits in phenomena such as intermittency and pruning, reduce the number of periodic orbits required for accurate eigenspectra computations, provide useful cross checks of the accuracy of trace formulas and cycle expansions, might lead to replacement of periodic orbit sums by contour integrals, and provide error bounds on approximations of smooth flows by normal form mappings. Still, the explicit sum rules for simple models presented above are only a modest step in direction of moving "beyond periodic orbit theory":

(a) The results presented depend on either the finiteness of polynomials (for the contour integral method) or properties of integers (for the Farey map case). One needs to develop infinite dimensional versions of the above finite rank operators L (ref. [11] is a step in this direction).

(b) One needs to reinstate positive measures. While in quantum mechanical applications the signed measures might be just the right Maslov phases, in the classical context the above sum rules are essentially index theorems and play a role analogous to what Lefschetz zeta function plays vis-a-vis the Artin-Mazur counting zeta function. (Appendix of ref. [9] is a step in this direction). 
(c) The transfer operator weights studied here were restricted to powers of the cycle stability; one needs to deal with more general classes of weighted operators.

\section{Acknowledgments}

Authors are grateful to $\mathrm{HH}$ Rugh for bringing ref. 442 to their attention, G Anderson [43] for kindly sending us his unpublished notes with a different proof of the rationality of the Hatjispiros-Vivaldi zeta functions, and to G Tanner for a critical reading of an early version of the manuscript. The transfer matrices (28) for the quadratic case were derived by $\mathrm{KH}$ as a part of the Niels Bohr Institute 3rd year undergraduate physics project. PC is grateful to the Isaac Newton Institute, Cambridge, and Dj. Cvitanović, Kostrena, where significant parts of this work was done, for kind hospitality, and the Carlsberg Fundation for many years of unflagging support. JR gratefully acknowledges financial support by the Studienstiftung des deutschen Volkes. GV thanks the support of the Hungarian Science Foundation OTKA (F019266/F17166/T17493) and the Hungarian Ministry of Culture and Education FKFP 0159/1997.

\section{Appendix A. Examples of sum rules}

To give the reader a feeling for what the above sum rules mean in practice, we shall work out explicitly the few simplest examples. The quadratic polynomial fixed points and their stabilities are given by

$$
\begin{aligned}
& x_{0}=(1+\sqrt{1+4 c}) / 2, \quad x_{1}=(1-\sqrt{1+4 c}) / 2, \\
& \Lambda_{0}=2 x_{0}, \quad \Lambda_{1}=2 x_{1},
\end{aligned}
$$

and the 2-cycle stability by

$$
\Lambda_{01}=4(1-c) .
$$

Appendix A.1. Trace formula for $\mathcal{L}_{(0)}$

The sum rule (17) states that for the quadratic polynomial (22)

$$
0=\frac{1}{\Lambda_{0}-1}+\frac{1}{\Lambda_{1}-1}, \quad 0=\frac{1}{\Lambda_{0}^{2}-1}+\frac{1}{\Lambda_{1}^{2}-1}+\frac{2}{\Lambda_{01}-1},
$$

and so on. This sum rule is not new; it goes back to Fatou [44] and Julia [45] and can be found, for example, in sect. 3.5 of ref. [15], proven by manipulating roots of polynomials rather than by the contour integration method. The Fredholm determinant (20) is in this case trivial, $F(z, 0)=1$. 


\section{Appendix A.2. Trace formula for $\mathcal{L}_{(1)}$}

The sum rule (18) for the quadratic polynomial fixed points and 2-cycles can be checked by hand:

$$
2=\frac{\Lambda_{0}}{\Lambda_{0}-1}+\frac{\Lambda_{1}}{\Lambda_{1}-1}, \quad 4=\frac{\Lambda_{0}^{2}}{\Lambda_{0}^{2}-1}+\frac{\Lambda_{1}^{2}}{\Lambda_{1}^{2}-1}+2 \frac{\Lambda_{01}}{\Lambda_{01}-1} .
$$

For quadratic polynomials $N=2^{n}$, so both the Fredholm determinant 20) and the dynamical zeta function (30) are given by $F(z, 1)=1-2 z, 1 / \zeta_{(1)}=F(z, 1) / F(z, 0)=$ $1-2 z$. For the case of all periodic points real, the traces $T_{n}(1)$ count the numbers of periodic points of period $n$, and $1 / \zeta_{(1)}$ is the Artin-Mazur topological zeta function 446.

Appendix A.3. Trace formula for $\mathcal{L}_{(2)}$

The sum rule (19) for the quadratic polynomial is given by

$$
\sum_{i \in \operatorname{Fix}\left(f^{n}\right)} \frac{\Lambda_{i}^{2}}{\Lambda_{i}-1}=4^{n},
$$

the Fredholm determinant by $F(z, 2)(z)=1-4 z$, and the dynamical zeta function by

$$
1 / \zeta_{(2)}=\frac{F(z, 2)}{F(z, 1)}=\frac{1-4 z}{1-2 z} .
$$

Furthermore, as for every $k>0$ the term (10) contributes a factor $\left(1-2^{k} z\right)$ to $F(z, k)$, $1 / \zeta_{(k)}$ always contains a factor $\left(1-2^{k} z\right) /\left(1-2^{k-1} z\right)$, in agreement with ref. [10].

\section{Appendix A.4. Trace formula for $\mathcal{L}_{(3)}$}

According to (14), we only need to to evaluate $C(3)$ for the quadratic polynomial

$$
C(3)=\oint \frac{d x}{2 \pi i} \frac{8 x^{3}}{x^{2}-c}=8 \oint \frac{d x}{2 \pi i} x\left(1+\frac{c}{x^{2}}+\ldots\right)=8 c .
$$

Together with (21) this yields the $T_{n}(3)$ sum rule for the quadratic polynomial

$$
\sum_{i \in \operatorname{Fix}\left(f^{n}\right)} \frac{\Lambda_{i}^{3}}{\Lambda_{i}-1}=8^{n}+(8 c)^{n} .
$$

For example, it is easily verified that

$$
\begin{aligned}
& 8(1+c)=\frac{\Lambda_{0}^{3}}{\Lambda_{0}-1}+\frac{\Lambda_{1}^{3}}{\Lambda_{1}-1} \\
& 64\left(1+c^{2}\right)=\frac{\Lambda_{0}^{6}}{\Lambda_{0}^{2}-1}+\frac{\Lambda_{0}^{6}}{\Lambda_{1}^{2}-1}+2 \frac{\Lambda_{01}^{3}}{\Lambda_{01}-1} .
\end{aligned}
$$

The Fredholm determinant is given by

$$
F(z, 3)(z)=(1-8 z)(1-8 c z)
$$


in agreement with the $k=3$ case of our general formula (27). The dynamical zeta function is

$$
1 / \zeta_{(3)}=\frac{F(z, 3)}{F(z, 2)}=\frac{(1-8 z)(1-8 c z)}{1-4 z} .
$$

Thus the first "nontrivial" zeta function is indeed of the form conjectured in ref. [10].

\section{Appendix A.5. Trace formula for $\mathcal{L}_{(7)}$}

We finish this appendix by writing out explicitely a typical nontrivial example: $k=7=2 \times 3+1$. According to (28)

$$
\mathbf{L}_{(7)}=\left[\begin{array}{ccc}
c^{3} & 3 c & 0 \\
c^{4} & 6 c^{2} & 1 \\
c^{5} & 10 c^{3} & 5 c
\end{array}\right] .
$$

The corresponding Fredholm determinant is given in table 1 This, as well as any other $F(z, k)$ that we have computed agrees with the form conjectured in ref. [10].

\section{References}

[1] D. Ruelle, Statistical Mechanics, Thermodynamic Formalism, (Addison-Wesley, Reading MA, 1978).

[2] M.C. Gutzwiller, Chaos in Classical and Quantum Mechanics (Springer, New York 1990).

[3] P. Cvitanović, ed., Periodic Orbit Theory - theme issue, CHAOS 2, 1-158 (1992).

[4] T. Tél and E. Ott, eds., Chaotic Scattering - theme issue, CHAOS 3, 417-782 (1993).

[5] G. Casati and B. Chirikov, eds., Quantum Chaos (Cambridge University Press, Cambridge 1994).

[6] N.J. Balmforth, P. Cvitanović, G.R. Ierley, E.A. Spiegel and G. Vattay, "Advection of vector fields by chaotic flows" Proceed. 8th Florida Workshop in Nonlinear Astronomy: Noise (New York Academy of Sciences, New York 1993).

[7] A.É. Eremenko and G.M. Levin, 'Periodic points of polynomials', Ukrain. Mat. J. 41, 1258 (1989), [English translation].

[8] G. Levin, M. Sodin, and P. Yuditskii, 'Ruelle operators with rational weights for Julia sets', J. d'Analyse Math. 63, 303 (1994).

[9] A. Eremenko, G. Levin, and M. Sodin, 'On the distribution of zeros of a Ruelle zeta-function', Comm. Math. Phys. 159, 433 (1994).

[10] S. Hatjispiros and F. Vivaldi, "A family of rational zeta functions for the quadratic map", Nonlinearity 8, 321 (1995).

[11] P. Contucci and A. Knauf, "The phase transition of the number-theoretical spin chain", Forum Mathematicum 9, 547 (1997).

[12] P. Cvitanović, P.E. Rosenqvist, H.H. Rugh, and G. Vattay, "A Fredholm determinant for semiclassical quantization", CHAOS 3, 619 (1993).

[13] Classical and Quantum Chaos - Periodic Orbit Theory, P. Cvitanović, R. Artuso, R. Mainieri, G. Vatay et al., http://www.nbi.dk/ predrag/QCcourse/, textbook in preparation.

[14] R. Artuso, E. Aurell and P. Cvitanović, "Recycling of strange sets I: Cycle expansions", Nonlinearity 3, 325 (1990).

[15] R.L. Devaney, An Introduction to Chaotic Dynamical Systems (Addison-Wesley, Reading MA, 1987).

[16] T. Bohr, P. Cvitanović and M.H. Jensen, Europhys. Letts. 6, 445 (1988). 
[17] H.-O. Peitgen, H. Jürgens and D. Saupe, Chaos and Fractals (Springer Verlag 1992).

[18] J. Rolf, "Comparison between cycle expansion and adjoint equations", http://www.nbi.dk/ChaosBook/projects/rolf.ps.gz (1997).

[19] P. Cvitanović, "Circle Maps: Irrationally Winding", in C. Itzykson, P. Moussa and M. Waldschmidt, eds., Number Theory and Physics, Les Houches 1989 Spring School, (Springer, New York 1992).

[20] M.J. Feigenbaum, Nonlinearity 1, 577 (1988).

[21] P. Cvitanović, G.H. Gunaratne and M.J. Vinson, Nonlinearity 3, 873 (1990).

[22] T.C. Halsey, M.H. Jensen, L.P. Kadanoff, I. Procaccia and B.I. Shraiman, Phys. Rev. A107, 1141 (1986).

[23] R. Artuso, P. Cvitanović and B.G. Kenny, Phys. Rev. A39, 268 (1989).

[24] G. T. Williams and D. H. Browne, Amer. Math. Monthly 54, 534 (1947).

[25] R.S. MacKay, doctoral thesis (Princeton University, 1982).

[26] P. Cvitanović and J. Myrheim, Phys. Lett. A94, 329 (1983); Commun. Math. Phys. 121, 225 (1989)

[27] P. Cvitanović, B. Shraiman and B. Söderberg, Physica Scripta 32, 263 (1985).

[28] M.J. Feigenbaum, J. Stat. Phys. 52, 527 (1988).

[29] A. Knauf, "On a ferromagnetic spin chain", Commun. Math. Phys. 153, 77 (1993).

[30] D.H. Mayer, Commun. Math. Phys. 130, 311 (1990).

[31] R. Artuso, E. Aurell and P. Cvitanović, "Recycling of strange sets II: Applications", Nonlinearity 3, 361 (1990).

[32] Y. Jiang, T. Morita and D. Sullivan, "Expanding direction of the period doubling operator", Comm. Math. Phys. 144, 509 (1992).

[33] F. Christiansen, P. Cvitanović and H.H. Rugh, "The spectrum of the period-doubling operator in terms of cycles", J. Phys A 23, L713 (1990).

[34] M. Pollicott, "A Note on the Artuso-Aurell-Cvitanović approach to the Feigenbaum tangent operator", J. Stat. Phys., 62, 257 (1991).

[35] P. Cvitanović, Phys. Rev. Lett. 61, 2729 (1988).

[36] D. Ruelle, Ergod. The. and Dynam. Sys. 2, 99 (1976).

[37] H.H. Rugh, "The Correlation Spectrum for Hyperbolic Analytic Maps", Nonlinearity 5, 1237 (1992).

[38] M. Kuš, F. Haake and D. Delande, "Prebifurcation periodic ghost orbits in semi-classical quantization", Phys. Rev. Lett. 71, 2167 (1993).

[39] G. Vattay, A. Wirzba and P.E. Rosenqvist, "Periodic Orbit Theory of Diffraction", Phys. Rev. Lett. 73, 2304 (1994).

[40] J. H. Hannay and A. M. Ozorio de Almeida, J. Phys. A 17, 3429, (1984).

[41] S.F. Nielsen et al., "Exact sum rules for billiards", in preparation.

[42] G. Levin, M. Sodin and P. Yuditskii, "A Ruelle operator for a real Julia set", Comm. Math. Phys. 141, 119 (1991).

[43] G. Anderson, Notes on dynamical zeta functions, U. of Minessota preprint, (March 1995).

[44] P. Fatou, Bull. Soc. Math. France 47, 161 (1919); ibid. 48, 33 and 208 (1920).

[45] G. Julia, J. Math. Pures et Appl. 4, 47 (1918).

[46] E. Artin and B. Mazur, Annals. Math. 81, 82 (1965). 